\title{
Cognitive Function and the Risk of Death in Chronic Kidney Disease
}

\author{
Kalani L. Raphael ${ }^{a, b}$ Guo Wei $^{\text {b }}$ Tom Greene $^{a, b}$ Bradley C. Baird ${ }^{b}$ \\ Srinivasan Beddhu ${ }^{a, b}$ \\ ${ }^{\text {a } V A ~ H e a l t h c a r e ~ S y s t e m, ~ a n d ~}{ }^{b}$ Department of Internal Medicine, University of Utah School of Medicine, \\ Salt Lake City, Utah, USA
}

\section{Key Words}

Cognitive function $\cdot$ Cognitive score $\cdot$ Chronic kidney

disease $\cdot$ Mortality

\begin{abstract}
Background and Aims: Cognitive impairment is a risk factor for death in dialysis patients and the general population. We sought to determine if cognitive impairment is associated with death in people with non-dialysis-dependent chronic kidney disease (CKD), and if so, whether this relationship is greater in the CKD population compared to the general population. Methods: National Health and Nutrition Examination Survey-III participants older than 60 years were asked to subtract 3 from 20 five times and to perform immediate and delayed recall of three items. A cognitive score of $0-11$ was assigned based on the number of correct responses. Participants were categorized according to cognitive score (11, 9-10, 6-9, and 0-5) and CKD status. Survival analyses were conducted using Cox models. Results: Within the CKD subpopulation, those in the lowest cognitive score group had a twofold increased hazard of death compared to those with maximum score. Within the non-CKD subpopulation, those in the lowest cognitive score group had a $46 \%$ increased hazard of death compared to those with maximum score. However, the difference in the hazards of death in the CKD and non-CKD subpopulations with the lowest cognitive score
\end{abstract}

was not significant ( $p=0.99$ ). Conclusions: Low cognitive score is associated with an increased risk of death in elderly individuals with and without CKD; however, there was no interaction of CKD and low cognitive score in this analysis.

Copyright $\odot 2011$ S. Karger AG, Basel

\section{Introduction}

Cognitive impairment is common in people with chronic kidney disease (CKD) $[1,2]$ and end-stage renal disease (ESRD) [3]. People with CKD have lower cognitive scores [4] and higher rates of cognitive decline than those without CKD $[4,5]$. Cognitive impairment is also associated with the severity of kidney disease [6-9], with the greatest cognitive impairment observed in those with ESRD [10]. Cognitive impairment is associated with an increased risk of death in individuals with ESRD [11] and is an independent risk factor for death in the general population [12-16]. To the best of our knowledge, the relationship between cognitive impairment and death in people with non-dialysis-dependent CKD has not been characterized, nor has the strength of the relationship between cognitive function and death been compared between individuals with and without CKD. Therefore, using data from the Third National Health and Nutrition Examination Survey (NHANES III), we investigated

\section{KARGER}

(c) 2011 S. Karger AG, Basel

Fax +4161306 1234

E-Mail karger@karger.ch

www.karger.com
Accessible online at:

www.karger.com/ajn
Kalani L. Raphael, MD

85 North Medical Drive East

Room 201

Salt Lake City, UT 84112 (USA)

Tel. +1 801585 3810, E-Mail kalani.raphael@ hsc.utah.edu 
whether a relationship between poor cognitive function and death exists among individuals with non-dialysisdependent CKD, and if so, whether the strength of this relationship differs among individuals with and without CKD.

\section{Materials and Methods}

\section{Study Population}

NHANES III was a cross-sectional survey of the noninstitutionalized, civilian population of the US conducted between 1988 and 1994 [17]. A complex, multistage sampling design was employed so that results could be extrapolated to represent the general US population as of the early 1990s [18]. Participants were included in this analysis if they were older than 60 years, completed the age-appropriate cognitive assessment at the time of the home interview, and had estimated glomerular filtration rate (eGFR) $\leq 150 \mathrm{ml} / \mathrm{min} / 1.73 \mathrm{~m}^{2}$. A random sample of participants between the ages of 20 and 59 years received a different cognitive assessment than individuals older than 60 years [19] and were not included in this study.

\section{Cognitive Assessment and Scoring}

Cognition was assessed in participants older than 60 years using an abbreviated version of the Mini-Mental State Examination (MMSE) $[17,20]$. Trained personnel asked participants to serially subtract 3 from 20 five times and to perform immediate and delayed recall of 3 items (table, apple, and penny). A score of 1 was assigned for each correct response. Thus, the range of possible scores was $0-11$.

\section{Baseline Data Ascertainment}

Trained personnel gathered baseline medical information. Diabetes was defined as self-reported history of diabetes, use of oral hypoglycemic agents or insulin, or fasting plasma glucose $\geq 126$ $\mathrm{mg} / \mathrm{dl}$. Hypertension was defined as systolic blood pressure $>140$ $\mathrm{mm} \mathrm{Hg}$ and/or diastolic blood pressure $>90 \mathrm{~mm} \mathrm{Hg}$, current treatment for hypertension, or if the participant was ever told to take medication for high blood pressure or she/he had hypertension. Physical inactivity was defined as no reported leisure time physical activity.

eGFR was estimated by the 4-variable Modification of Diet in Renal Disease equation $[175 \times$ (standardized serum creatinine $)^{-1.154} \times(\text { age })^{-0.203} \times 0.742$ (if female) $\times 1.212$ (if AfricanAmerican)] using standardized serum creatinine [21]. Serum creatinine was measured using a kinetic rate Jaffe method and recalibrated to standardized creatinine measurements obtained at the Cleveland Clinic Research Laboratory (Cleveland, Ohio, USA) as standardized creatinine $=-0.184+0.960 \times$ NHANES III measured serum creatinine [22]. CKD was defined as an eGFR $<60$ $\mathrm{ml} / \mathrm{min} / 1.73 \mathrm{~m}^{2}$. Individuals with eGFR $60-150 \mathrm{ml} / \mathrm{min} / 1.73 \mathrm{~m}^{2}$ were classified as not having CKD. Those with eGFR $>150 \mathrm{ml} /$ $\mathrm{min} / 1.73 \mathrm{~m}^{2}$ were excluded from this analysis. Serum and plasma specimens from collection sites were transported on dry ice to the central laboratories and stored at $-70^{\circ} \mathrm{C}$ until analysis. Serum 25 -hydroxyvitamin D level was measured by a radioimmunoassay (RIA) using INCSTAR Corporation 25-Hydroxyvitamin D
${ }^{125}$ I RIA kit (Stillwater, Minn., USA). Serum C-reactive protein (CRP) was measured by latex-enhanced nephelometry using a Behring Nephelometer Analyzer System using reagents from Behring Diagnostics Inc. (Somerville, N.J., USA).

\section{Follow-Up Data}

The National Center for Health Statistics created a NHANES III Linked Mortality File that contains mortality follow-up data from the date of NHANES III survey participation. This information was based upon the results from a probabilistic match between NHANES III and National Death Index death certificate records, the details of which are provided elsewhere [23]. Mortality data up until December 31, 2000 were used in this analysis.

\section{Statistical Analyses}

We used the svy suite of commands in Stata 11 (Stata 11, College Station, Tex., USA) and followed the analytical guidelines for NHANES data proposed by the Centers for Disease Control [18]. It should be noted that the svy suite of commands in Stata use the survey design elements of NHANES to calculate the expected means and proportions of the entire US, non-institutionalized, civilian population. Based on the distribution of the cognitive scores, 4 cognitive score groups were categorized: maximum score of 11 (57.1\%), score of 9-10 (23.4\%), score of 6-8 (14.0\%), and score of $0-5(5.5 \%)$. Distributions of baseline characteristics across the four cognitive groups were examined with $\chi^{2}$ contingency table analysis for categorical variables and analysis of variance for continuous variables; both using the svy set of commands in Stata 11.

\section{Analyses of Low Cognitive Score in the CKD and Non-CKD \\ Subpopulations}

Logistic regression was used to relate the odds of a low cognitive score $(0-5)$ to the presence of CKD first in an unadjusted analysis followed by adjustment for demographic factors (age, gender, race, education, and income) history of cardiovascular disease (myocardial infarction, congestive heart failure, and stroke), hypertension, diabetes, physical inactivity, smoking, and alcohol use.

A multivariable logistic regression model was used to examine potential factors associated with low cognitive score $(0-5)$ in the CKD and non-CKD subpopulations. Demographic factors, history of cardiovascular disease, hypertension, diabetes, physical inactivity, smoking, alcohol use, eGFR, serum 25-hydroxyvitamin D, CRP, hemoglobin, and urinary albumin to creatinine levels were included in the model. CRP and urinary albumin to creatinine levels were log-transformed to better approximate normality since these variables were highly skewed.

\section{Survival Analyses}

Cox models were used to examine the association between cognitive score and death. Eight groups were categorized based on the presence or absence of CKD and the four cognitive score groups (11, 9-10, 6-8 and 0-5). The hazard of death was determined in the eight groups, with the non-CKD and cognitive score of 11 group serving as the reference group in unadjusted (model 1) and adjusted models. Adjustments were made for demographic factors, history of cardiovascular disease, hypertension, diabetes, physical inactivity, smoking, alcohol use, and albuminuria (model 2). Model 3 was further adjusted for serum CRP, serum 25-hy- 
Table 1. Baseline characteristics of the study population according to cognitive score

\begin{tabular}{|c|c|c|c|c|c|}
\hline & \multicolumn{4}{|l|}{ Cognitive score } & \multirow[t]{2}{*}{$\mathrm{p}$ value } \\
\hline & $11(57.1 \%)$ & $9-10(23.4 \%)$ & $6-8(14.0 \%)$ & $0-5(5.5 \%)$ & \\
\hline \multicolumn{6}{|l|}{ Demographics } \\
\hline Age, years ${ }^{1}$ & $68.7 \pm 0.3$ & $71.4 \pm 0.4$ & $72.5 \pm 0.4$ & $75.4 \pm 0.6$ & $<0.001$ \\
\hline Men, $\%$ & $45(43-48)$ & $47(43-50)$ & $36(31-40)$ & $42(38-47)$ & 0.003 \\
\hline African-American race, $\%$ & $5(4-6)$ & $7(6-9)$ & $15(12-19)$ & $24(19-29)$ & $<0.001$ \\
\hline Education $<12$ years, $\%$ & $30(26-24)$ & $45(41-50)$ & $64(59-69)$ & $79(72-85)$ & $<0.001$ \\
\hline Family income USD <20,000/year, \% & $38(34-41)$ & $54(48-59)$ & $68(62-73)$ & $77(68-84)$ & $<0.001$ \\
\hline \multicolumn{6}{|l|}{ Clinical characteristics } \\
\hline Myocardial infarction, \% & $10(8-12)$ & $13(11-15)$ & $11(8-14)$ & $17(12-23)$ & 0.005 \\
\hline Congestive heart failure, $\%$ & $6(5-8)$ & $6(5-8)$ & $8(6-11)$ & $12(8-18)$ & 0.017 \\
\hline Stroke, \% & $5(4-6)$ & $8(6-10)$ & $7(5-10)$ & $13(10-16)$ & $<0.001$ \\
\hline Diabetes, $\%$ & $15(12-17)$ & $16(14-19)$ & $18(14-22)$ & $24(19-31)$ & 0.008 \\
\hline Hypertension, \% & $61(58-63)$ & $65(61-70)$ & $67(62-71)$ & $65(58-72)$ & 0.078 \\
\hline $\mathrm{CKD}, \%$ & $17(15-19)$ & $23(20-27)$ & $26(23-30)$ & $25(22-29)$ & $<0.001$ \\
\hline Physical inactivity, \% & $18(15-20)$ & $22(19-26)$ & $32(29-35)$ & $48(41-54)$ & $<0.001$ \\
\hline Current smoker, $\%$ & $16(14-19)$ & $13(10-16)$ & $16(13-21)$ & $14(9-19)$ & 0.204 \\
\hline Current alcohol use, $\%$ & $40(35-45)$ & $30(26-36)$ & $19(14-26)$ & $16(11-22)$ & $<0.001$ \\
\hline \multicolumn{6}{|l|}{ Laboratory data } \\
\hline Urine albumin:creatinine, $\mathrm{mg} / \mathrm{g}^{2}$ & $8.3(4.6-17.7)$ & $9.0(5.0-21.7)$ & $10.6(5.6-25.4)$ & $12.7(5.9-37.7)$ & 0.13 \\
\hline Hemoglobin, g/dl ${ }^{1}$ & $14.0 \pm 0.1$ & $14.0 \pm 0.1$ & $13.7 \pm 0.1$ & $13.4 \pm 0.1$ & $<0.001$ \\
\hline Serum $25-\mathrm{OH}$ vitamin $\mathrm{D}, \mathrm{ng} / \mathrm{ml}^{1}$ & $28.0 \pm 0.3$ & $28.4 \pm 0.5$ & $25.9 \pm 0.4$ & $25.1 \pm 0.8$ & $<0.001$ \\
\hline $\mathrm{CRP}, \mathrm{mg} / \mathrm{l}^{2}$ & $2.1(2.1-4.8)$ & $2.1(2.1-5.3)$ & $2.1(2.1-6.2)$ & $2.1(2.1-5.9)$ & 0.26 \\
\hline
\end{tabular}

Values are shown as percentages $(95 \% \mathrm{CI}) .{ }^{1}$ Presented as mean \pm standard error. ${ }^{2}$ Presented as median and interquartile range.

droxyvitamin D, and hemoglobin levels. Assumptions of proportional hazards were examined by comparing the logarithm of the hazard ratio for each predictor variable in the first 3 years of follow-up to the logarithm of the hazard ratio of the predictor variables after 3 years. Cognitive function groups did not violate proportionality assumptions. Gender and history of stroke were the only factors that violated proportionality assumptions and were included as stratification variables instead of covariables.

Cox analyses were next performed in the CKD and non-CKD subpopulations, separately. Within each subpopulation, the hazard ratio of death was determined for each cognitive score group compared to those with score of 11. Similar adjustments were made as in the previous Cox analysis, except that eGFR was included as a covariate in models 2 and 3. Likewise, gender and history of stroke were stratification variables in these models.

Two methods were used to examine if there was an interaction of CKD with low cognitive score and death. In each subpopulation, the association of cognitive score with mortality was analyzed as a continuous variable after adjusting for demographic factors, history of cardiovascular disease, hypertension, diabetes, physical inactivity, smoking, alcohol use, eGFR, and albuminuria in separate Cox models. Regression coefficients from each Cox model were compared using a large sample normal approximation. In the second, we used a large-sample $\chi^{2}$ approximation with 3 degrees of freedom to compare the log hazard ratios corresponding to the cognitive score categories between the CKD and non-CKD subpopulations.

Cognitive Function, Mortality and CKD

\section{Results}

\section{Baseline Characteristics}

Of the 20,050 NHANES III participants, 5,086 (25.4\%) were older than 60 years, underwent the cognitive assessment, and had eGFR $<150 \mathrm{ml} / \mathrm{min} / 1.73 \mathrm{~m}^{2}$. Table 1 presents characteristics of these participants according to cognitive score $(11,9-10,6-8$, and $0-5)$. As expected, those with the lowest cognitive score were older. This group also had a greater proportion of individuals who were African-American, received $<12$ years of education, had an annual family income USD $<20,000$, had diabetes and cardiovascular diseases, and had no leisure physical activity. A lower percentage of participants in the low cognitive score group consumed alcohol at the time of participation as well. Lower eGFR, hemoglobin, and serum 25-hydroxyvitamin D levels were also observed in those with the lowest cognitive score (table 1).

In this group of participants older than 60 years, $21.5 \%$ had CKD. Figure 1 presents the percent of individuals within each cognitive score group according to CKD status. A lower percentage of individuals in the CKD subpopulation achieved the maximum score of 11 and a 


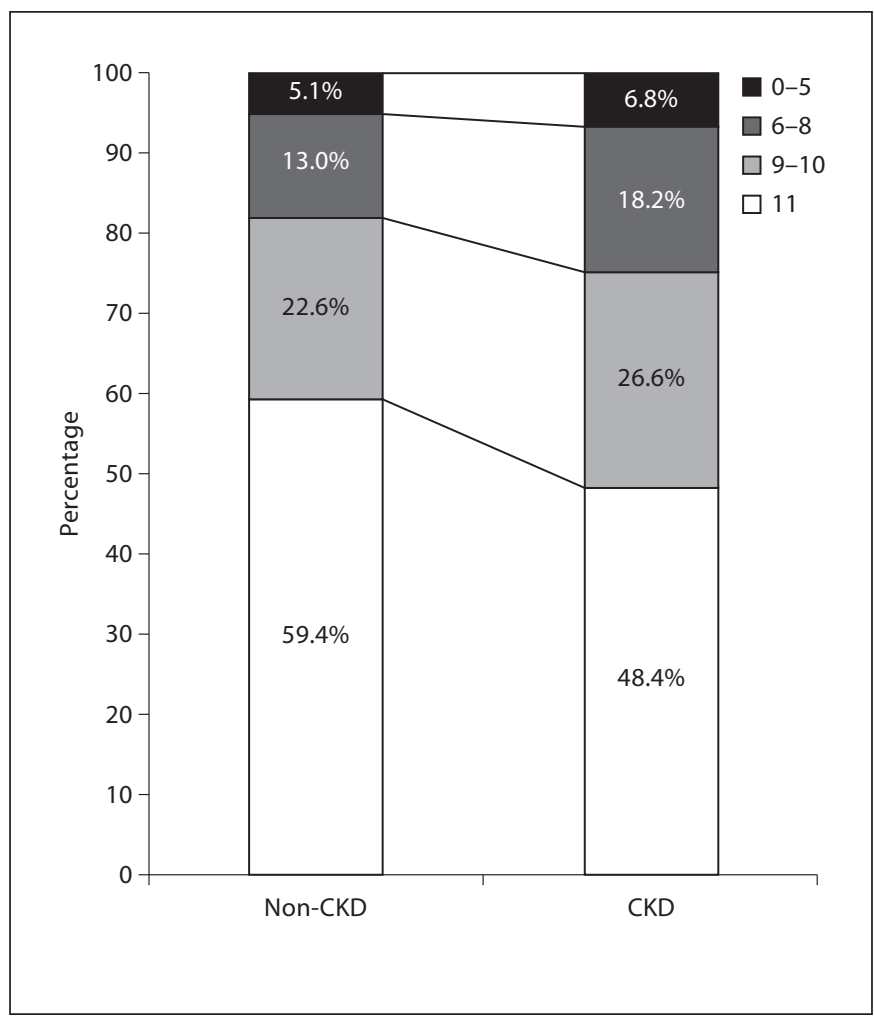

Fig. 1. Stacked bar graph of the percentages of NHANES III participants older than 60 years of age in each cognitive score group in the non-CKD and CKD subpopulations. A lower percentage of those with CKD achieved the maximum cognitive score (11). Values for the non-CKD subpopulation do not add up to $100 \%$ due to rounding.

higher percentage were in the lowest cognitive score category in comparison to the non-CKD subpopulation.

The unadjusted odds of low cognitive score in the CKD subpopulation were 1.36 (95\% CI 1.09-1.71) compared to the non-CKD subpopulation. However, this association became non-significant (OR 1.06, 95\% CI 0.62 1.81) after adjusting for demographic factors, history of cardiovascular disease, hypertension, diabetes, physical inactivity, smoking, and alcohol use. In a sensitivity analysis, the odds of less than maximum score $(<11)$ were not different between the CKD and non-CKD subpopulations after identical adjustment.

\section{Factors Associated with Low Cognitive Score}

Physical inactivity, less than 12 years of education, and African-American race were associated with low cognitive score (0-5) in both non-CKD and CKD subpopulations. Increasing age, male gender, annual family income
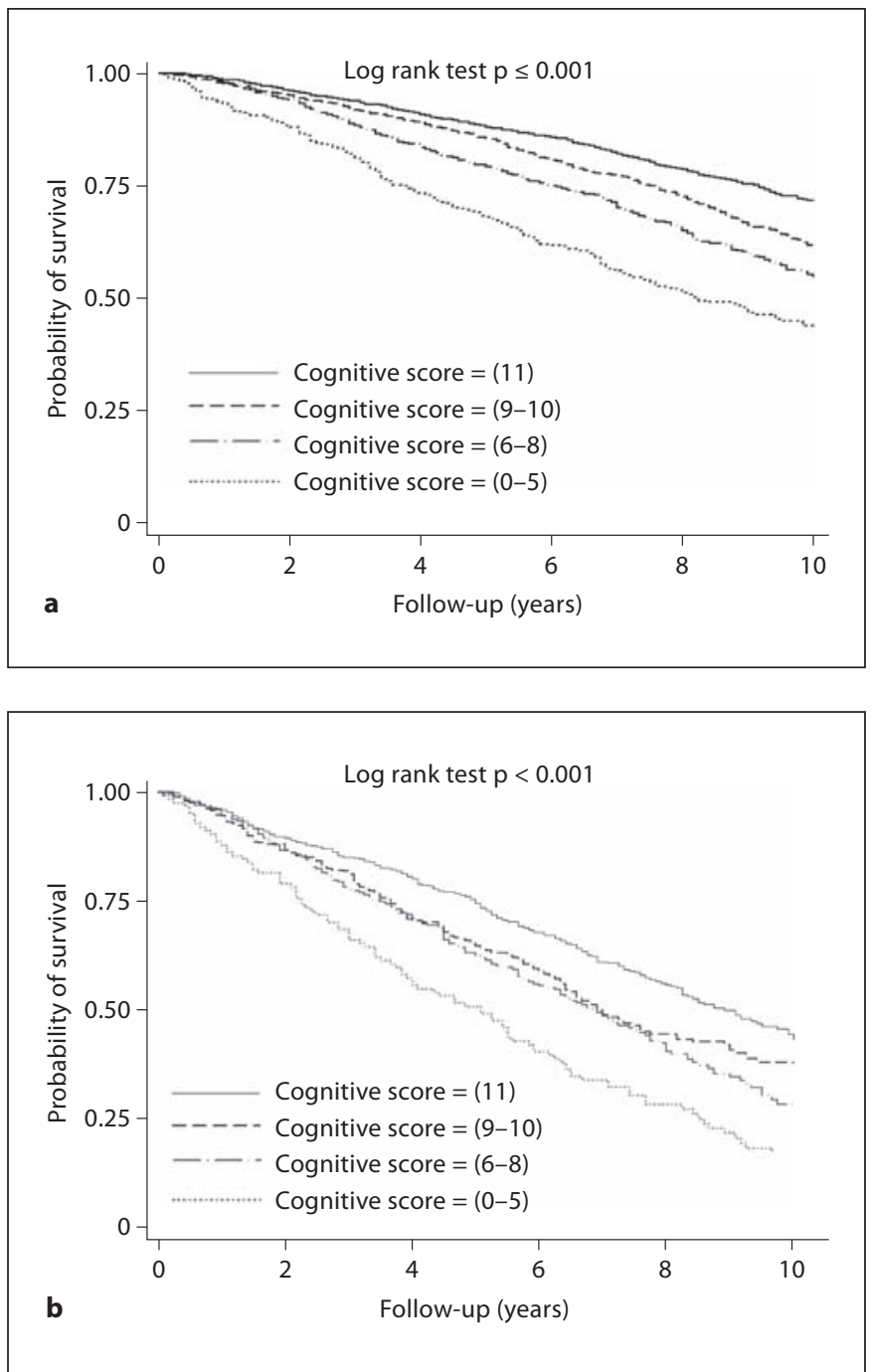

Fig. 2. Kaplan-Meier plots of the unadjusted probability of survival by cognitive score in the non-CKD subpopulation (a) and CKD subpopulation (b).

USD $<20,000$, and history of myocardial infarction were additional factors associated with lower cognitive score in the non-CKD subpopulation (table 2).

\section{Survival Analyses}

The unadjusted probability of survival was progressively lower among those groups with lower cognitive scores in both the non-CKD and CKD subpopulations (fig. 2). Table 3 presents results from Cox regression models in which the hazards of death were determined in subgroups partitioned by CKD status and cognitive score. Those without CKD and a score of 11 served as the refer- 
Table 2. Multivariable logistic regression models of the odds of low cognitive score $(0-5)$ in the non-CKD and CKD subpopulations

\begin{tabular}{|c|c|c|c|c|}
\hline & \multicolumn{2}{|c|}{ Non-CKD subpopulation } & \multicolumn{2}{|c|}{ CKD subpopulation } \\
\hline & OR & $95 \% \mathrm{CI}$ & OR & $95 \% \mathrm{CI}$ \\
\hline \multicolumn{5}{|l|}{ Demographic } \\
\hline Each additional year in age & 1.08 & $1.04-1.11$ & 1.05 & $0.98-1.12$ \\
\hline Male vs. female & 1.94 & $1.28-2.96$ & 1.19 & $0.45-3.14$ \\
\hline African-American race vs. Caucasian & 2.81 & $1.69-4.69$ & 2.74 & $1.25-6.04$ \\
\hline Education $(<12$ years $)$ & 3.13 & $1.89-5.21$ & 7.23 & $2.52-20.7$ \\
\hline Family income (USD <20,000/year) & 1.90 & $1.09-3.31$ & 1.41 & $0.66-3.02$ \\
\hline \multicolumn{5}{|l|}{ Clinical and laboratory variables } \\
\hline Myocardial infarction & 2.11 & $1.08-4.14$ & 0.37 & $0.15-0.93$ \\
\hline Stroke & 1.44 & $0.73-2.84$ & 0.48 & $0.16-1.44$ \\
\hline Congestive heart failure & 0.94 & $0.37-2.41$ & 1.07 & $0.44-2.60$ \\
\hline Diabetes & 1.41 & $0.77-2.58$ & 0.68 & $0.29-1.59$ \\
\hline Hypertension & 0.73 & $0.41-1.29$ & 1.44 & $0.51-4.06$ \\
\hline Physical inactivity & 1.78 & $1.12-2.84$ & 2.46 & $1.31-4.63$ \\
\hline Current alcohol use & 0.97 & $0.60-1.59$ & 0.75 & $0.28-1.99$ \\
\hline History of smoking & 1.32 & $0.68-2.56$ & 0.53 & $0.18-1.57$ \\
\hline Each $\mathrm{ml} / \mathrm{min} / 1.73 \mathrm{~m}^{2}$ increase in eGFR & 1.01 & $0.99-1.02$ & 1.00 & $0.97-1.03$ \\
\hline Each doubling of urinary ACR, mg/g & 1.10 & $0.94-1.28$ & 1.02 & $0.84-1.24$ \\
\hline Each $\mathrm{ng} / \mathrm{ml}$ increase in serum $25-\mathrm{OH}$ vitamin $\mathrm{D}$ & 1.00 & $0.97-1.03$ & 0.98 & $0.95-1.01$ \\
\hline Each $\mathrm{g} / \mathrm{dl}$ increase in hemoglobin & 0.81 & $0.67-0.99$ & 1.00 & $0.80-1.24$ \\
\hline Each doubling of CRP, $\mathrm{mg} / \mathrm{l}$ & 0.94 & $0.81-1.09$ & 0.92 & $0.73-1.16$ \\
\hline
\end{tabular}

$\mathrm{ACR}=$ Albumin to creatinine ratio.

ence group. Compared to those without CKD and with a cognitive score of 11, all other subgroups had a higher hazard of death in unadjusted analyses (table 3). After adjusting for demographic factors, history of cardiovascular disease, hypertension, diabetes, physical inactivity, smoking, alcohol use, and albuminuria, with gender and history of stroke as stratification variables, the hazard ratios of death for all other subgroups were greater than the reference group (table 3, model 2). The hazard ratio of death was 2.84 for those in the CKD and lowest cognitive score group compared to the reference group. Further adjustment for CRP, hemoglobin, and serum 25-hydroxyvitamin $\mathrm{D}$ level minimally attenuated these hazard ratios (table 3, model 3).

Similar Cox analyses were performed separately for the non-CKD and CKD subpopulations (table 4 and fig. 3) to analyze the relationship between cognitive score and death within these subgroups. In unadjusted analyses, the hazard ratios of death were greater with progressively lower cognitive scores in the non-CKD and CKD subpopulations. This dose-response relationship between cognitive score and mortality persisted in each subpopulation after adjustment for demographic factors, history of cardiovascular disease, hypertension, diabetes, physical inactivity, smoking, alcohol use, eGFR, and albuminuria with stratification by gender and history of stroke. In the low cognitive score groups, the hazards of death were 1.46 in the non-CKD subpopulation $(95 \%$ CI $1.07-2.01)$ and 2.03 in the CKD subpopulation (95\% CI 1.16-3.55) compared to those with cognitive score of 11 in the same subpopulation.

Using cognitive score as a continuous variable in Cox analyses, the hazards of death were 0.96 (95\% CI $0.92-$ 1.01) in the non-CKD subpopulation and 0.93 (95\% CI $0.87-1.01$ ) after adjustment for demographic factors, history of cardiovascular disease, hypertension, diabetes, physical inactivity, smoking, alcohol use, eGFR, and albuminuria with stratification by gender and history of stroke. The difference between the Cox regression coefficients in the CKD and non-CKD subpopulations was not significant when the cognitive score was treated as a continuous variable $(p=0.99)$ or when it was categorized as in table $4(\mathrm{p}=0.99)$. 
Table 3. Cox regression models of the hazards of death according to CKD status and cognitive score (CS) group

\begin{tabular}{llll}
\hline & Model 1 & Model 2 & Model 3 \\
\hline Non-CKD and CS $=11$ & reference & reference & reference \\
Non-CKD and CS $=9-10$ & $1.51(1.23-1.85)$ & $1.22(1.00-1.48)$ & $1.19(0.98-1.45)$ \\
Non-CKD and CS $=6-8$ & $2.06(1.64-2.59)$ & $1.31(0.97-1.76)$ & $1.21(0.91-1.62)$ \\
Non-CKD and CS $=0-5$ & $3.56(2.67-4.73)$ & $1.64(1.23-2.20)$ & $1.55(1.14-2.10)$ \\
CKD and CS $=11$ & $2.35(1.82-3.04)$ & $1.73(1.36-2.20)$ & $1.58(1.23-2.03)$ \\
CKD and CS $=9-10$ & $3.36(2.56-4.41)$ & $1.77(1.24-2.52)$ & $1.62(1.13-2.31)$ \\
CKD and CS $=6-8$ & $3.85(2.78-5.32)$ & $1.91(1.37-2.66)$ & $1.81(1.31-2.51)$ \\
CKD and CS $=0-5$ & $8.38(6.19-11.34)$ & $2.84(1.70-4.75)$ & $2.59(1.52-4.41)$ \\
\hline
\end{tabular}

Model 1: Unadjusted.

Model 2: Adjusted for demographic factors, history of cardiovascular diseases, hypertension, diabetes, physical inactivity, history of smoking, alcohol use, and albuminuria; stratified by gender and history of stroke.

Model 3: Model 2 variables plus CRP, hemoglobin, and serum 25-hydroxyvitamin D; stratified by gender and history of stroke.

Table 4. Cox regression models of the hazards of death in the non-CKD and CKD subpopulations according to CS

\begin{tabular}{llll}
\hline & Model 1 & Model 2 & Model 3 \\
\hline $\begin{array}{lll}\text { Non-CKD subpopulation } \\
\text { CS }=11\end{array}$ & reference & reference & reference \\
CS $=9-10$ & $1.51(1.24-1.86)$ & $1.20(0.99-1.47)$ & $1.19(0.98-1.45)$ \\
CS $=6-8$ & $2.07(1.64-2.60)$ & $1.25(0.93-1.68)$ & $1.18(0.89-1.56)$ \\
CS $=0-5$ & $3.58(2.69-4.77)$ & $1.46(1.07-2.01)$ & $1.39(1.00-1.93)$ \\
CKD subpopulation & & & \\
CS $=11$ & reference & reference & reference \\
CS $=9-10$ & $1.41(1.03-1.92)$ & $1.16(0.83-1.62)$ & $1.14(0.80-1.62)$ \\
CS $=6-8$ & $1.62(1.16-2.26)$ & $1.24(0.85-1.80)$ & $1.24(0.85-1.80)$ \\
$C S=0-5$ & $3.47(2.53-4.75)$ & $2.03(1.16-3.55)$ & $2.02(1.11-3.67)$ \\
\hline
\end{tabular}

Model 1: Unadjusted.

Model 2: Adjusted for demographic factors, history of cardiovascular disease, hypertension, diabetes, physical inactivity, smoking, alcohol use, eGFR and albuminuria; stratified by gender and history of stroke.

Model 3: Model 2 variables plus CRP, hemoglobin, and serum 25-hydroxyvitamin D; stratified by gender and history of stroke.

\section{Discussion}

In this study, the cognitive assessment administered to NHANES III participants older than 60 years during the home examination was used to test the hypothesis that poorer cognition is associated with an increased hazard of death in older individuals with non-dialysis-dependent CKD. Compared to those without CKD and maximum cognitive score, those with CKD and low cognitive score $(0-5)$ had a nearly threefold increased hazard of death. As expected, CKD itself explains a portion of this increased hazard since those with CKD and maximum cognitive score had a 73\% increased hazard of death compared to those without CKD and maximum cognitive score. Still, within the CKD subpopulation, those with low cognitive score had a twofold increased hazard of death compared to those with maximum score. Therefore, the results of this study suggest that poor cognitive score among older individuals with non-dialysis-dependent CKD is associated with an increased risk of death, 


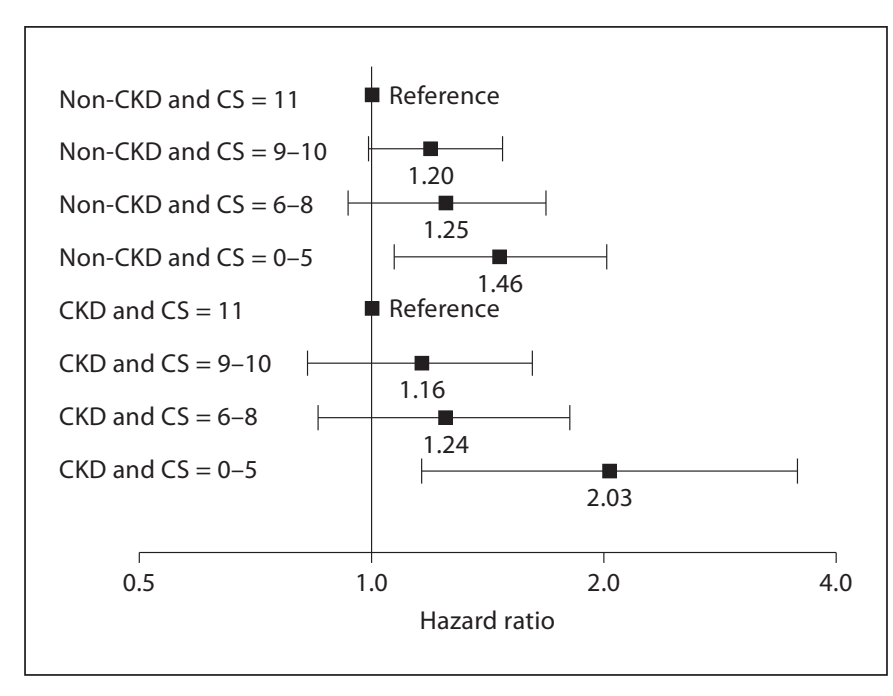

Fig. 3. Hazard of death according to cognitive score (CS) within the non-CKD and CKD subpopulations after adjusting for demographic factors, history of cardiovascular disease, hypertension, diabetes, physical inactivity, smoking, alcohol use, eGFR and albuminuria; stratified by gender and history of stroke. Separate Cox models were performed for each subpopulation. Those with the maximum cognitive score of 11 serve as the reference group within each subpopulation.

as it is in the general population and those with ESRD. Similarly, low cognitive score was associated with a $46 \%$ increased hazard of death compared to those with maximum score in the non-CKD subpopulation, consistent with previous studies that have observed an association between cognitive impairment and an increased hazard of death in the general population [12-16].

We also sought to test the hypothesis that individuals with CKD and lower cognitive score have a greater risk of death than those without CKD and similar cognitive score. Using the cognitive assessment in NHANES III, lower cognitive score was associated with an increased hazard of death in the CKD and non-CKD subpopulations; however, there was no difference in the hazards of death between the CKD and non-CKD subpopulations. These results suggest that there is no interaction effect of CKD on the relationship between low cognitive score and mortality, even though people with CKD have an overall greater risk of death compared to those without CKD.

Furthermore, after adjusting for demographic factors, history of cardiovascular disease, hypertension, diabetes, physical inactivity, smoking, and alcohol use, the CKD subpopulation did not have higher odds of having either low cognitive score $(0-5)$ or less than maximum cogni- tive score $(<11)$ compared to those without CKD. Thus, even though cognitive impairment is common in people with CKD, we were unable to detect a differential risk of having poor cognitive score among NHANES participants with and without CKD that was independent of these factors.

The reasons for an overall increased risk of death among individuals with CKD and lower cognitive score in this study are speculative. As in the general population, cognitive impairment might lead to non-adherence with medications [30] or increased risk of accidents or falls [31] in those with CKD, which may account for the increased risk of death observed. Other studies have found associations between cognitive impairment and metabolic syndrome [32], inflammation [33-35], insulin resistance [36], muscle strength [37], physical inactivity [38], and subsequent dementia [39]. However, adjusting for several of these factors did not attenuate the hazard of death in individuals with CKD and low cognitive score.

The major strength of this study is that it was conducted using data from a large cohort of individuals assembled to represent the non-institutionalized, civilian US population. Furthermore, the cognitive test used components of the MMSE [20], which is routinely administered in clinical practice. Assessing cognitive function using this abbreviated form of the MMSE in the clinic might be a simple and rapid way to identify individuals at increased risk of death.

Like any observational study, unmeasured residual confounding needs to be considered while interpreting the results. Also, we can only infer that the relationships in this analysis identify associations and not causative pathways. The cognitive function test was an abbreviated version of the MMSE [20]. Hence, it is possible that those with the maximum possible score on the NHANES III administered cognitive assessment might still have cognitive impairment, potentially leading to misclassification bias. A different cognitive test was performed in NHANES III participants older than 60 years during the examination. However, because there were $>1,000$ fewer observations during the examination than the home interview, we restricted the analysis to the home-administered cognitive assessment. The attention, calculation, and memory components in this abbreviated version of the MMSE are unable to detect deficiencies in other cognitive domains such as executive function; hence, the relationship between other cognitive domains and death in CKD could not be investigated in this analysis. Since just 69 of the 1,094 NHANES III participants with CKD had stage IV CKD, we cannot rule out the possibility that an 
interaction of cognitive function and death might be present in people with more advanced predialysis CKD. Additionally, these results are only applicable to individuals older than 60 years. As the NHANES III population consisted of non-institutionalized civilians, institutionalized individuals with dementia are excluded from this study. This might have underestimated the association of cognitive function and mortality in CKD. Finally, depression could potentially influence performance on the test. Depression could not be accounted for in this analysis because adults older than 40 years of age were not assessed for depression in NHANES III.

In summary, an inverse relationship between cognitive score and death was observed in older individuals with and without CKD; however, there is no interaction effect of CKD on this relationship.

\section{Acknowledgements}

This investigation is supported by the following: National Institutes of Health and the National Cancer Institute grant 1 KM1CA156723; National Institutes of Health Office of the Director grant 5TL1RR025762-03; National Kidney Foundation of Utah grant awarded to K.L.R.; NIDDK grants RO1-DK077298 and RO1-DK078112 awarded to S.B.; The University of Utah Study Design and Biostatistics Center, with funding in part from the Public Health Services research grant numbers UL1-RR025764 and C06-RR11234 from the National Center for Research Resources.

\section{Disclosure Statement}

None of the authors has any conflicts of interest to report.

\section{References}

1 Elias MF, Elias PK, Seliger SL, Narsipur SS, Dore GA, Robbins MA: Chronic kidney disease, creatinine and cognitive functioning. Nephrol Dial Transplant 2009;24:24462452.

2 Madan P, Kalra OP, Agarwal S, Tandon OP: Cognitive impairment in chronic kidney disease. Nephrol Dial Transplant 2007;22: 440-444.

-3 Murray AM, Tupper DE, Knopman DS, Gilbertson DT, Pederson SL, Li S, Smith GE, Hochhalter AK, Collins AJ, Kane RL: Cognitive impairment in hemodialysis patients is common. Neurology 2006;67:216-223.

4 Khatri, M, Nickolas T, Moon YP, Paik MC, Rundek T, Elkind MS, Sacco RL, Wright CB: CKD associates with cognitive decline. J Am Soc Nephrol 2009;20:2427-2432.

-5 Buchman, AS, Tanne D, Boyle PA, Shah RC, Leurgans SE, Bennett DA: Kidney function is associated with the rate of cognitive decline in the elderly. Neurology 2009;73:920927.

-6 Etgen T, Sander D, Chonchol M, Briesenick C, Poppert H, Forstl H, Bickel H: Chronic kidney disease is associated with incident cognitive impairment in the elderly: the INVADE study. Nephrol Dial Transplant 2009;24:3144-3150.

7 Kurella M, Chertow GM, Fried LF, Cummings SR, Harris T, Simonsick E, Satterfield $\mathrm{S}$, Ayonayon H, Yaffe K: Chronic kidney disease and cognitive impairment in the elderly: the health, aging, and body composition study. J Am Soc Nephrol 2005;16:2127-2133.
8 Kurella Tamura M, Wadley V, Yaffe K, McClure LA, Howard G, Go R, Allman RM, Warnock DG, McClellan W: Kidney function and cognitive impairment in US adults: the Reasons for Geographic and Racial Differences in Stroke (REGARDS) Study. Am J Kidney Dis 2008;52:227-234.

-9 Yaffe K, Ackerson L, Kurella Tamura M, Le Blanc P, Kusek JW, Sehgal AR, Cohen D, Anderson C, Appel L, Desalvo K, Ojo A, Seliger S, Robinson N, Makos G, Go AS: Chronic kidney disease and cognitive function in older adults: findings from the chronic renal insufficiency cohort cognitive study. J Am Geriatr Soc 2010;58:338-345.

10 Kurella M, Chertow GM, Luan J, Yaffe K: Cognitive impairment in chronic kidney disease. J Am Geriatr Soc 2004;52:18631869.

11 Kurella M, Mapes DL, Port FK, Chertow GM: Correlates and outcomes of dementia among dialysis patients: the Dialysis Outcomes and Practice Patterns Study. Nephrol Dial Transplant 2006;21:2543-2548.

12 Dewey, ME, Saz P: Dementia, cognitive impairment and mortality in persons aged 65 and over living in the community: a systematic review of the literature. Int J Geriatr Psychiatry 2001;16:751-761.

13 Fried LP, Kronmal RA, Newman AB, Bild DE, Mittelmark MB, Polak JF, Robbins JA, Gardin JM: Risk factors for 5-year mortality in older adults: the Cardiovascular Health Study. JAMA 1998;279:585-592.

14 Lee HB, Kasper JD, Shore AD, Yokley JL, Black BS, Rabins PV: Level of cognitive impairment predicts mortality in high-risk community samples: the memory and medical care study. J Neuropsychiatry Clin Neurosci 2006;18:543-546.
15 Sampson EL, Bulpitt CJ, Fletcher AE: Survival of community-dwelling older people: the effect of cognitive impairment and social engagement. J Am Geriatr Soc 2009;57:985991.

16 Shavelle RM, Paculdo DR, Strauss DJ, Kush SJ: Cognitive impairment and mortality in the Cardiovascular Health Study. J Insur Med 2009;41:110-116.

17 National Center for Health Statistics: Plan and Operation of The Third National Health and Nutrition Examination Survey, 19881994. Hyattsville, National Center for Health Statistics, 1995

18 National Center for Health Statistics: Analytical and Reporting Guidelines: The Third National Health and Nutrition Examination Survey, 1988-1994. Hyattsville, National Center for Health Statistics, 1996.

19 Hailpern SM, Melamed ML, Cohen HW, Hostetter TH: Moderate chronic kidney disease and cognitive function in adults 20 to 59 years of age: Third National Health and Nutrition Examination Survey (NHANES III). J Am Soc Nephrol 2007; 18:2205-2213.

20 Folstein MF, Robins LN, Helzer JE: The Mini-Mental State Examination. Arch Gen Psychiatry 1983;40:812.

-21 Levey AS, Coresh J, Greene T, Stevens LA, Zhang YL, Hendriksen S, Kusek JW, Van Lente F: Using standardized serum creatinine values in the modification of diet in renal disease study equation for estimating glomerular filtration rate. Ann Intern Med 2006;145:247-254 
-22 Selvin E, Manzi J, Stevens LA, Van Lente F, Lacher DA, Levey AS, Coresh J: Calibration of serum creatinine in the National Health and Nutrition Examination Surveys (NHANES) 1988-1994, 1999-2004. Am J Kidney Dis 2007;50:918-926.

23 National Center for Health Statistics: The Third National Health and Nutrition Examination Survey (NHANES III) Linked Mortality File: Matching Methodology. Hyattsville, National Center for Health Statistics, 2005.

-24 Wood RY, Giuliano KK, Bignell CU, Pritham WW: Assessing cognitive ability in research: use of MMSE with minority populations and elderly adults with low education levels. J Gerontol Nurs 2006;32:45-54.

-25 Murden RA, McRae TD, Kaner S, Bucknam ME: Mini-Mental State exam scores vary with education in blacks and whites. J Am Geriatr Soc 1991;39:149-155.

-26 Kemoun G, Thibaud M, Roumagne N, Carette P, Albinet C, Toussaint L, Paccalin M, Dugue B: Effects of a physical training programme on cognitive function and walking efficiency in elderly persons with dementia. Dement Geriatr Cogn Disord 2010;29: 109-114.

-27 Lautenschlager NT, Cox KL, Flicker L, Foster JK, van Bockxmeer FM, Xiao J, Greenop KR, Almeida OP: Effect of physical activity on cognitive function in older adults at risk for Alzheimer disease: a randomized trial. JAMA 2008;300:1027-1037.
28 Venturelli M, Lanza M, Muti E, Schena F: Positive effects of physical training in activity of daily living-dependent older adults. Exp Aging Res 2010;36:190-205.

29 Pitkala KH, Raivio MM, Laakkonen ML, Tilvis RS, Kautiainen H, Strandberg TE: Exercise rehabilitation on home-dwelling patients with Alzheimer's disease - a randomized, controlled trial. Study protocol. Trials 2010;11:92.

30 Hayes TL, Larimer N, Adami A, Kaye JA: Medication adherence in healthy elders: small cognitive changes make a big difference. J Aging Health 2009;21:567-580.

31 Liu-Ambrose TY, Ashe MC, Graf P, Beattie $\mathrm{BL}$, Khan KM: Increased risk of falling in older community-dwelling women with mild cognitive impairment. Phys Ther 2008; 88:1482-1491.

32 Panza F Frisardi V, Capurso C, Imbimbo BP Vendemiale G, Santamato A, D’Onofrio G, Seripa D, Sancarlo D, Pilotto A, Solfrizzi V: Metabolic syndrome and cognitive impairment: current epidemiology and possible underlying mechanisms. J Alzheimers Dis 2010;21:691-724.

33 Roberts RO, Geda YE, Knopman DS, Boeve BF, Christianson TJ, Pankratz VS, Kullo IJ, Tangalos EG, Ivnik RJ, Petersen RC: Association of C-reactive protein with mild cognitive impairment. Alzheimers Dement 2009;5:398-405
34 Roberts RO, Geda YE, Knopman DS, Cha RH, Boeve BF, Ivnik RJ, Pankratz VS, Tangalos EG, Petersen RC: Metabolic syndrome, inflammation, and nonamnestic mild cognitive impairment in older persons: a population-based study. Alzheimer Dis Assoc Disord 2010;24:11-18.

35 Xu G, Zhou Z, Zhu W, Fan X, Liu X: Plasma $\mathrm{C}$-reactive protein is related to cognitive deterioration and dementia in patients with mild cognitive impairment. J Neurol Sci 2009;284:77-80.

>36 Bruehl H, Sweat V, Hassenstab J, Polyakov V, Convit A: Cognitive impairment in nondiabetic middle-aged and older adults is associated with insulin resistance. J Clin Exp Neuropsychol 2010;32:487-493.

37 Boyle PA, Buchman AS, Wilson RS, Leurgans SE, Bennett DA: Association of muscle strength with the risk of Alzheimer disease and the rate of cognitive decline in community-dwelling older persons. Arch Neurol 2009;66:1339-1344.

38 Masel MC, Raji M, Peek MK: Education and physical activity mediate the relationship between ethnicity and cognitive function in late middle-aged adults. Ethn Health 2010; 15:283-302.

39 Petersen RC, Doody R, Kurz A, Mohs RC, Morris JC, Rabins PV, Ritchie K, Rossor M, Thal L, Winblad B: Current concepts in mild cognitive impairment. Arch Neurol 2001;58: 1985-1992. 